\title{
O MECANISMO DO DESEMPENHO DE POLI-2- MERCAPTOBENZIMIDAZOL NA DETECÇÃO ELETROQUÍMICA DE COLESTEROL
}

\author{
V. Tkach $^{1}$, M.R.M. Hosseini ${ }^{2}$, R. Ojani ${ }^{3}$, O. Yelenich ${ }^{1}$, P.I. Yagodynets' ${ }^{1}$ \\ nightwatcher2401@gmail.com
}

${ }^{1}$ Universidade Nacional de Chernivtsi, Ucrânia

${ }^{2}$ Universidade Iraniana de Ciência e Tecnologia, Teerã, República Islâmica do Irã

${ }^{3}$ Universidade de Mazandarã, Babolsar, República Islâmica do Irã

\section{RESUMO}

O desempenho de poli-2-mercaptobenzimidazol na detecção eletroquímica de colesterol foi avaliado fenomenológica e matematicamente. O modelo matemático correspondente foi desenvolvido e analisado por meio de teoria de estabilidade lineal e análise de bifurcações. Os dados teóricos foram comparados com os experimentais e com os teóricos para os sistemas análogos.

Palavras-chave: colesterol, polímeros condutores, sensores eletroquímicos, poli2-mercaptobenzimidazol, estado estacionário estável

\section{INTRODUÇÃO}

Colesterol [1] é um álcool policíclico, derivado de ciclopentanperidrofenantreno, considerado um esteroide do grupo de esteróis. É precursor de hormônios sexuais [2] de animais vertebrados (inclusive o homem), bem como de hormônio de mudança de cor dos insetos. Toma parte ativa do metabolismo.

No entanto, haja vista a sua insolubilidade n'água, o seu excesso pode provocar o bloqueio de corrente sanguínea [3], bem como problemas renais e de fígado. Destarte, a procura de um método eficiente da sua detecção é uma tarefa atual [4 - 8].

A utilização de elétrodos quimicamente modificados é um dos métodos modernos, utilizados na química eletroanalítica contemporânea [9-12]. Neste caso, um modificador usado (como polímero condutor, complexo, corante, ou outra) é escolhido 
haja vista a sua compatibilidade com o analito (segundo o princípio de chave e fechadura). No entanto, há dificuldades no seu uso com o colesterol, haja vista os problemas da previsão de mecanismo mais provável do desempenho de um sensor hipotético, bem como o existirem instabilidades eletroquímicas durante a eletrooxidação de compostos orgânicos (inclusive a eletrossíntese de polímeros condutores) [13 - 21], o que pode prejudicar a interpretação do sinal analítico.

Os dois problemas, bem como o da comparação com os sistemas análogos, empregando outros analitos e outros materiais [22 - 35] sem ensaios experimentais, resolvem-se pelo desenvolvimento de um modelo matemático, capaz de descrever adequadamente o desempenho do sistema eletroanalítico, o que se fará neste trabalho para colesterol como analito e poli(2-mercaptobenzimidazol) como substância modificadora [12].

\section{O SISTEMA E O SEU MODELO}

No sensor, descrito em [12], foi descrita a eletropolimerização de 2mercaptobenzimidazol na presença de colesterol, que se acompanhava pelo entrapment das suas moléculas. Não foi, de fato, um processo químico - as moléculas do hormônio ficaram presas entre as moléculas do polímero condutor sem poder sair da sua "matriz". Este processo, controlado pela difusão de mercaptobenzimidazol e do hormônio, e obedecerá, em termos gerais, aos modelos matemáticos, expostos em [36 - 37], levemente modificados (por conta do acréscimo de um adendo que descreve a saída do analito da matriz polimérica).

No caso da ausência de analito, o comportamento durante a eletropolimerização obedecerá a um dos modelos, descritos em [38 - 42] (dependendo das condições da síntese), sendo o 2-mercaptobenzimidazol o monômero.

Para descrever a eletrodetecção de colesterol, por meio da sua imobilização por dopagem da matriz polimérica, introduzir-se-ão as três variáveis:

c - a concentração de colesterol na camada pré-superficial;

$\sigma-$ o grau de dopagem da matriz polimérica pelo colesterol;

$\theta$ - o grau de revestimento da parte do polímero, quimicamente modificada pelo analito.

É possível provar que, haja vista as suposições, feitas, por exemplo, em [36 42], o conjunto de equações diferenciais que descreve este sistema se expõe como: 


$$
\left\{\begin{array}{c}
\frac{d c}{d t}=\frac{2}{\delta}\left(\frac{\Delta}{\delta}\left(c_{0}-c\right)+d_{-1}-d_{1}\right) \\
\frac{d \sigma}{d t}=\frac{1}{\Sigma}\left(d_{1}-d_{-1}-r_{1}\right) \\
\frac{d \theta}{d t}=\frac{1}{G}\left(r_{1}-r_{2}\right)
\end{array}\right.
$$

Em que $\Delta$ é coeficiente de difusão, $\delta$ é espessura da camada pré-superficial, $\mathrm{d}_{1} \mathrm{e}$ d-1, a velocidade de dopagem-desdopagem da matriz pelo analito, $\Sigma$ é o número máximo de sítios de dopagem, fornecidos pela matriz do polímero condutor revestido, G é a concentração máxima do polímero condutor, r1 e r2 são as reações de modificações química e eletroquímica do polímero condutor.

As velodicadades das respectivas reações podem calcular-se como:

$$
\begin{gathered}
d_{1}=k_{d 1} c(1-\sigma) \exp \left(\alpha_{1} \sigma\right) \\
d_{-1}=k_{d-1} \sigma \exp \left(-\alpha_{2} \sigma\right) \\
r_{1}=\kappa_{1} \sigma(1-\theta) \\
r_{2}=\kappa_{2} \theta \exp \left(\frac{n F \varphi_{0}}{R T}\right)
\end{gathered}
$$

em que os parâmetros $\mathrm{k}$ são constantes de respectivas reações, $\alpha_{1}$ e $\alpha_{2}$ descrevem as interações entre o dopante e a matriz, $\mathrm{n}$ é o número de elétrons transferidos, F é o número de Faraday, $\varphi_{0}$ é o salto do potencial na dupla camada elétrica (DCE), relacionado ao da carga zero, $R$ é a constante universal de gases e T, a temperatura absoluta do vaso.

A principal diferença deste sistema dos relacionados [22 - 35] é a presença de uma etapa preliminar de dopagem-dedopagem do analito pelo polímero condutor, anterior à sua reação específica. Isto dá impacto significante ao comportamento do sistema.

\section{RESULTADOS E DISCUSSÃO}

Para analisar o comportamento do sistema da eletrodetecção de colesterol por meio da sua imobilização na matriz de 2-mercaptobenzimidazol, analisamos o conjunto de equações diferenciais (1), haja vista as relações algébricas (2 - 5) por meio da teoria de estabilidade linear. A matriz funcional de Jacobi, cujos elementos são calculados para o estado estacionário, vê-se como:

$$
\left(\begin{array}{lll}
a_{11} & a_{12} & a_{13} \\
a_{21} & a_{22} & a_{23} \\
a_{31} & a_{32} & a_{33}
\end{array}\right)
$$


em que:

$$
\begin{array}{ll}
a_{11}=\frac{2}{\delta}\left(-\frac{\Delta}{\delta}-k_{d 1}(1-\sigma) \exp \left(\alpha_{1} \sigma\right)\right) & a_{12}= \\
\frac{2}{\delta}\left(k_{d-1} \exp \left(-\alpha_{2} \sigma\right)-\alpha_{2} k_{d-1} \sigma \exp \left(-\alpha_{2} \sigma\right)+k_{d 1} \exp \left(\alpha_{1} \sigma\right)-\alpha_{1} k_{d 1}(1-\right. & \\
\left.\sigma) \exp \left(\alpha_{1} \sigma\right)\right) & \\
a_{13}=0 & a_{22}= \\
a_{21}=\frac{1}{\Sigma}\left(k_{d 1}(1-\sigma) \exp \left(\alpha_{1} \sigma\right)\right) & \\
\frac{1}{\Sigma}\left(-k_{d-1} \exp \left(-\alpha_{2} \sigma\right)+\alpha_{2} k_{d-1} \sigma \exp \left(-\alpha_{2} \sigma\right)-k_{d 1} \exp \left(\alpha_{1} \sigma\right)+\alpha_{1} k_{d 1}(1-\right. & \\
\left.\sigma) \exp \left(\alpha_{1} \sigma\right)-\kappa_{1}(1-\theta)\right) & \\
a_{23}=\frac{1}{\Sigma}\left(\kappa_{1} \sigma\right) a_{31}=0 & \\
a_{32}=\frac{1}{G}\left(\kappa_{1}(1-\theta)\right) & \\
a_{33}=\frac{1}{G}\left(-\kappa_{1} \sigma-\kappa_{2} \exp \left(\frac{n F \varphi_{0}}{R T}\right)-\mathrm{u} \kappa_{2} \theta \exp \left(\frac{n F \varphi_{0}}{R T}\right)\right) &
\end{array}
$$

A instabilidade oscilatória neste caso é possível. Outrossim, é mais provável que nos sistemas típicos do desempenho eletroanalítico de polímeros condutores e materiais afins [22 - 35]. O porquê disso expor-se-á abaixo.

Para a bifurcação de Hopf se realizar, a diagonal principal da matriz de Jacobi deve ter elementos positivos, responsáveis pela positiva conexão de retorno.

Vê-se que, além do adendo $-\mathrm{u} \kappa_{2} \theta \exp \left(\frac{n F \varphi_{0}}{R T}\right)$, cuja positividade descreve as influências na DCE, causa comum do comportamento oscilatório nestes sistemas, outro elemento capazmente positivo é $\alpha_{2} k_{d-1} \sigma \exp \left(-\alpha_{2} \sigma\right)+\exp \left(\alpha_{1} \sigma\right)+\alpha_{1} k_{d 1}(1-$ $\sigma) \exp \left(\alpha_{1} \sigma\right)>0$, cuja positividade descreve a atração entre o analito-dopante, que é colesterol, e a matriz polimérica.

Confirma-se, destarte, a presença de estruturas dissipativas temporais, cuja existência se mantém pela difusão de colesterol (entrada de energia) e pela formação do polímero condutor na sua forma final (saída de energia).

Para analisar a estabilidade de estado estacionário, utilizamos o critério de RouthHurwitz, do qual se obtém o requisito geral Det $\mathrm{J}<0$. Para evitar a análise de expressões grandes, introduzimos as novas variáveis para o determinante da matriz aparecer como:

$$
\frac{2}{\delta \Sigma G}\left|\begin{array}{ccc}
-\kappa-\lambda & -X & 0 \\
\lambda & X-\Xi & -\Pi \\
0 & \Xi & \Pi-\gamma
\end{array}\right|
$$


Abrindo os parênteses, obter-se-á a condição de estabilidade do estado estacionário como:

$$
-\Pi \kappa X+\Upsilon \kappa X-\Gamma \kappa \Xi-\Upsilon \lambda \Xi<0
$$

Obteve-se uma expressão, típica para sistemas, em que a reação é regulada pela difusão, o que acontece, quando a dopagem é mais rápida, em grandes concentrações do analito. No entanto, sendo as concentrações do analito pequenas, a difusão passa a ser mais rápida que a dopagem e a reação reger-se-á pela dopagem.

Vê-se que a negatividade do parâmetro da dopagem-dedopagem $X$, descrevendo a fraqueza ou ausência de atração entre a matriz e o dopante e a positividade de $Y$ promovem a estabilidade do estado estacionário, porque deslocam a expressão à esquerda de (17) para valores mais negativos.

No caso da igualdade das influências desestabilizadoras às estabilizadoras, obtém-se a instabilidade monotônica, que aparece em:

$$
-\Pi \kappa X+\Upsilon \kappa X-\Gamma \kappa \Xi-\Upsilon \lambda \Xi=0
$$

Reação lateral efetiva entre o analito e o polímero, paralela à dopagem, acrescentará mais um adendo às equações (1) e (3). Tal reação dá seu impacto na estabilidade do estado estacionário, diminuindo a influência de dopagem.

Autocatálise para este polímero e analito não é possível, mas se houver com outro caso análogo, aparecerá como mais uma causa do comportamento oscilatório [22 - 35].

\section{CONCLUSÕES}

1. A presença da etapa preliminar de dopagem do analito pela matriz do polímero condutor faz o comportamento do sistema mais complicado, haja vista a presença, nele, dos nuances da dopagem.

2. O processo eletroanalítico é regulado pela difusão, o que acontece, quando a dopagem é mais rápida, em grandes concentrações do analito. No entanto, sendo as concentrações do analito pequenas, a difusão passa a ser mais rápida que a dopagem e a reação reger-se-á pela dopagem.

3. A instabilidade oscilatória, além das influências na CDE, pode ser causada, neste sistema, pela atração entre o analito-dopante e a matriz polimérica que o "hospeda".

4. Confirma-se a presença de estruturas dissipativas temporais, cuja existência se mantém pela difusão de colesterol (entrada de energia) e pela formação do polímero condutor na sua forma final (saída de energia).

\section{REFERÊNCIAS}


1. http://www.endocrino.org.br/colesterol/; acesso aos 8 de setembro de 2015

2. K. Zhang, R. Kaufmann, Nat. Cell. Biol., 5(2003), 769

3. http://www.nhs.uk/conditions/Cholesterol/Pages/Introduction.aspx ; acesso aos 8 de setembro de 2015

4. A. Mondal, N.R. Jana, Chem. Comm., 48(2012), 7316

5. T.T.N. Dinh, J.R.Blanton Jr., J.C. Brooks et. al., J. Food Comp. An., 21(2008), 306 
6. P. Sriwasadi, M.H. Kroll, P.H. Lolekha, Am. J. Clin. Path., 127(2007), 906

7. L.C. Bauer, D. de A. Santana, M. dos S. Macedo et. al., J. Braz. Chem. Soc., 25(2014), 161

8. C.M. Bandeira, J.M. Ferreira, G.C. Nogueira et. al., Quím. Nova., 36(2013), 1222

9. R. Ojani, V. Rahimi, J. Raoof, J. Chin. Chem. Soc., 62(2015), 90

10. S.E. Baghbamidi, H. Beitollahi, S. Tajik, Anal. Bioanal. Electrochem., 6(2014), 634

11. J.B. Raoof, R.Ojani, F. Jamali, S.R. Hosseini, Casp. J. Chem., 1(2012), 73

12. M.R. Milani Hosseini, Sh. Hemmati, A. Ghaffarinejad, A. Motaharian, Anal. Bioanal. Electrochem., 7(2015), 129

13. L. Hudson, M.R. Bassett, Rev. Chem. Eng, 7(1991), 108.

14. I.Das, N.R.Agrawal, S.A.Ansari, S.K.Gupta,Ind. J. Chem, 47(2008), 1798.

15. S.U. Rahman, M.S. Ba-Shammakh, Synth. Met. 140(2004), 207.

16. A.S.Liu, M.A.S. Oliveira. J. Braz. Chem Soc., 18(2007), 143.

17. Sazou D., Synth. Met. 130(2002), 45.

18. I.Das, N. Goel, N.R. Agrawal, S. K. Gupta, , J. Phys. Chem, 114(2010), 12888.

19. M. Bazzaoui, E.A. Bazzaoui, L. Martins, J.I. Martins, Synth. Met. 130(2002), 73.

20. I.Das, N. Goel, S. K. Gupta, N.R. Agrawal, J. Electroanal. Chem, 670(2012), 1

21. K.Aoki, I. Mukoyama, J.Chen, Russ.J. Electrochem, 40(2004), 319

22. V. Tkach, V. Nechyporuk, P. Yagodynets', Rev. Colomb. Cienc. Quím. Farm., 41(2012), 203

23. V.Tkach, V. Nechyporuk, P.Yagodynets, Rev. Colomb. Cien. Quím. Farm., 42(2013), 30

24. V.Tkach, V.Nechyporuk, P.Yagodynets', Anal. Bioanal. Electrochem., 6(2014), 273

25. V. Tkach, V. Nechyporuk, P. Yagodynets', Rev. Colomb. Cienc. Quím. Farm., 42(2013), 215

26. V. Tkach, V. Nechyporuk, P.Yagodynets', I. Rusnak, Orbital Elec. J. Chem, 4(2012), 136

27. V. Tkach V, V. Nechyporuk, P. Yagodynets', Yu. Meslyuk, Rev. Soc. Quím. Perú.,77(2011), 259

28. V. Tkach, H. Nascimento, V. Nechyporuk, P. Yagodynets', Rev. Col. Cienc. Quím. Farm., 43(2014), 196

29. V. Tkach, V. Nechyporuk, P. Yagodynets', III Encontro em Técnicas de Caracterização em Análise Química, 6 de junho de 2014, Braga, Portugal, P. 73

30. V. Tkach, R. Ojani, V. Nechyporuk, P. Yagodynets', IV International Conference of Young Scientists “Chemistry Today”, 18-22 of August, 2014, Yerevan, Armenia, P 49 
31. V. Tkach, V. Nechyporuk, R.Ojani, P.Yagodynets', M. Tazyrov. Anales del XXX Congreso Argentino de Química, 22 al 24 de Octubre de 2014, Buenos Aires., http://aqa.org.ar/pdf101/cd/Qca.Analitica/1-001.pdf, accessed at the 28th of February, 2015

32. V.Tkach, V.Nechyporuk e P. Yagodynets',Livro de Resumos do XVIII Encontro Luso-Galego de Química, 28 - 30 de Novembro 2012, Vila Real, Portugal, 29

33. V.V.Tkach, V.V. Nechyporuk, P.I. Yagodynets and Al. M. da Rocha, $2^{\text {nd }}$ International Conference "Advances in Heterocyclic Chemistry, 25-27 ${ }^{\text {th }}$ of September 2011, Tbilisi, Georgia, 126 - 127

34. V.Tkach, R. Ojani, V. Nechyporuk, P.Yagodynets, Rev. Colomb. Cien. Quím. Farm., 43(2014), 35

35. V. Tkach, R. Ojani, V. Nechyporuk, P. Yagodynets', Orbital. Elec. J. Chem., 6(2014), 142

36. V. Tkach, V. Nechyporuk., Ecl. Quím., 37(2012), 68

37. V. Tkach, V. Nechyporuk, P. Yagodynets', Afinidad., 70(2013), 73

38. V. Tkach, V. Nechyporuk, P. Yagodynets', Ciên. Tecn. Mat., 24(2012), 54

39. V. Tkach, V. Nechyporuk, P. Yagodynets', Med. J. Chem., 3(2015), 1122

40. V. Tkach, S. C. de Oliveira, O. I. Aksimentyeva et. al., Mor. J. Chem., 3(2015), 550

41. V. Tkach, V. Nechyporuk, P. Yagodynets', Quím. Bras., 7(2013), 59

42. V. Tkach, V. Nechyporuk, P. Yagodynets', Avan. Quím., 8(2013), 9 